\title{
Wells' Syndrome Mimicking Facial Cellulitis: A Report of Two Cases
}

\author{
Maxence Cormerais $^{\mathrm{a}}$ Florence Poizeau $^{\mathrm{a}}$ Laure Darrieux ${ }^{\mathrm{a}}$ \\ Laurent Tisseau $^{\text {b }}$ Gilles Safa ${ }^{a}$ \\ ${ }^{a}$ Department of Dermatology, Centre Hospitalier de Saint-Brieuc, Saint-Brieuc, and \\ ${ }^{\mathrm{b}}$ Armor Pathology Laboratory, Plérin, France
}

\section{Key Words}

Wells' syndrome $\cdot$ Eosinophilic cellulitis $\cdot$ Facial cellulitis

\begin{abstract}
Wells' syndrome (WS), or eosinophilic cellulitis, is an uncommon inflammatory dermatosis of unknown etiology that typically presents with pruritic cellulitis-like plaques on the extremities. Therefore, WS is often misdiagnosed as bacterial cellulitis due to its similarity in presentation. Here, we report two cases of WS that masqueraded as bacterial facial cellulitis. Under treatment with oral prednisone and/or a combination therapy with levocetirizine and hydroxyzine, both patients showed a dramatic improvement of the skin lesions. These cases highlight the need for clinicians to consider WS in the differential diagnosis when evaluating a patient with facial cellulitis that does not respond to an initial antimicrobial regimen. In addition, our cases suggest that combination therapy with levocetirizine and hydroxyzine may be successfully used as corticosteroid-sparing treatment or to prevent relapse after the discontinuation of corticosteroid treatment.

(C) 2015 S. Karger AG, Basel
\end{abstract}

\section{Introduction}

Wells' syndrome (WS), also known as eosinophilic cellulitis, is a rare inflammatory dermatosis of unknown etiology that typically presents with pruritic cellulitis-like plaques on the extremities. The typical histological features in the acute stage are characterized by dermal edema with a dense eosinophilic infiltrate without signs of vasculitis. In the subacute stage, distinctive flame figures are formed when degranulating eosinophils coat collagen bundles with eosinophilic major basic protein [1]. However, atypical presentations may oc- 
Cormerais et al.: Wells' Syndrome Mimicking Facial Cellulitis: A Report of Two Cases

cur. Here, we report two additional cases of WS that masqueraded as bacterial facial cellulitis.

\section{Case Reports}

Case 1

A healthy 31-year-old woman presented to the emergency department complaining of a painful, pruritic, facial erythematous edema covered by vesicles and yellowish crusts (fig. 1). The patient reported that the skin lesions had developed more than 12 days before her presentation. The general practitioner originally diagnosed her condition as bacterial facial cellulitis and prescribed oral antibiotics (pristinamycin and fusidic acid), which did not produce any improvement. The patient did not have any significant medical history and was not on any other medications. On physical examination, she was afebrile, with vital signs within normal limits, and general examination was normal. The following laboratory studies were within normal limits or negative: complete blood count with differential, C-reactive protein level, liver function tests, protein electrophoresis, antinuclear antibodies, serum electrolytes and urinalysis. The patient was admitted to the hospital with a diagnosis of bacterial facial cellulitis unresponsive to oral antibiotics and was treated with intravenous oxacillin. Over the following days, the erythema and swelling continued to progress despite intravenous antibiotic therapy. Bacterial blood and skin cultures were negative. A skin biopsy was performed and revealed a severe diffuse dermal infiltrate of eosinophils. No flame figures or signs of vasculitis were observed (fig. 2). Direct immunofluorescence was negative. Based on the clinical and histological findings, a diagnosis of WS was established. All antibiotic treatment was discontinued and the patient was subsequently started on $40 \mathrm{mg} /$ day oral prednisone with rapid improvement occurring within $48 \mathrm{~h}$. Prednisone was then tapered over 2 weeks with complete resolution of the skin lesions. After discontinuation of prednisone, the patient received maintenance therapy with a combination of $10 \mathrm{mg} /$ day levocetirizine and $50 \mathrm{mg} /$ day hydroxyzine and experienced no recurrence of disease during 12 months of follow-up.

\section{Case 2}

A 50-year-old woman with no significant medical history presented with acute onset of diffuse facial erythematous edema with vesiculobullous lesions (fig. 3) in association with fever $\left(38.8^{\circ} \mathrm{C}\right)$. The patient denied any drug intake and had an unremarkable general examination. Routine laboratory workup revealed a C-reactive protein level of $122 \mathrm{mg} / \mathrm{l}$ (normal $<5 \mathrm{mg} / \mathrm{l}$ ); other tests, including complete blood count with differential, liver function tests, protein electrophoresis, antinuclear antibodies, serum electrolytes and urinalysis, were normal or negative. The patient was admitted to the hospital with presumed bacterial facial cellulitis and was started on a course of intravenous clindamycin. Over the following 2 days, her skin lesions continued to worsen, and antibiotic coverage was broadened without improvement. Bacterial blood and skin cultures were negative. A skin biopsy was performed and revealed dermal edema and a dense dermal inflammatory infiltrate composed of eosinophils, neutrophils, lymphocytes and histiocytes without signs of vasculitis, suggesting the diagnosis of WS. All antibiotic treatments were discontinued and the patient was started on a combination therapy of $10 \mathrm{mg} /$ day levocetirizine and $50 \mathrm{mg} /$ day hydroxyzine. Within 3 days she became afebrile, the C-reactive protein level normalized, and the skin lesions dramatically improved (fig. 4). The patient was discharged and was instructed to continue 
Cormerais et al.: Wells' Syndrome Mimicking Facial Cellulitis: A Report of Two Cases

the combined antihistamine therapy to prevent recurrences. She remained under regular surveillance and did not show any sign of relapse during 3 months of follow-up.

\section{Discussion}

WS, or eosinophilic cellulitis, which was first described by Wells in 1971 [2], is a rare inflammatory dermatosis that is observed more commonly in adults than in children [3]. Although its etiology remains largely unknown, it is thought to be a hypersensitivity reaction to exogenous or endogenous stimuli. WS may be idiopathic or associated with infections, arthropod bites, hematological disorders, solid tumors or medications [1, 4]. In our patients, no triggering factor was clearly identified. Peripheral eosinophilia is present in approximately $50 \%$ of WS cases, and a correlation among the clinical activity of the disease, the levels of eosinophils in the blood and the serum interleukin-5 levels has been reported [5]. WS can also occur in association with Churg-Strauss syndrome, supporting the hypothesis that the two diseases might be part of the same pathogenic process [6].

There are several different clinical variants of WS, including urticarial-like, vesiculobullous, annular granuloma-like and papulonodular variants [7]. However, the classic cellulitis-like plaque variant is the most common. Therefore, WS is often misdiagnosed as bacterial cellulitis due to its similarity in presentation [8]. Indeed, in our cases, WS masqueraded as bacterial cellulitis of the face, leading to inappropriate empirical broad-spectrum antimicrobial treatment. In addition to clinical findings, a skin biopsy helps differentiate WS from infectious cellulitis. The characteristic histopathological findings include dermal edema, flame figures and a marked infiltrate of eosinophils into the dermis [1]. WS is usually a benign condition without systemic manifestations, although the clinical course may recur over several months or years. However, as was observed in case 2, WS patients may present with a variety of nonspecific symptoms, such as fever, arthralgia and malaise [8].

Systemic steroids are considered the first-line treatment for idiopathic WS. Topical corticosteroids, antihistamines, dapsone, cyclosporine and oral tacrolimus have also been used to treat WS, with variable success $[3,8]$. Interestingly, in our patients, combination therapy with levocetirizine and hydroxyzine produced a dramatic clearing of lesions in case 2 , and in case 1 this combined antihistamine therapy permitted long-term treatment to prevent relapses. To our knowledge, there has been only one reported case of WS successfully treated with cetirizine alone. This patient was a 12-year-old girl who developed recurrent pruritic papulonodular lesions of both legs. Cetirizine induced rapid response and permitted prolonged remission for 18 months [9]. Furthermore, previous studies have demonstrated that cetirizine, a metabolite of hydroxyzine, and levocetirizine inhibit transepidermal migration and chemotaxis of eosinophils [10].

In conclusion, these cases emphasize the importance of considering WS in the differential diagnosis when evaluating a patient with facial cellulitis that does not respond to an initial antimicrobial regimen. Misdiagnosis can lead to the inappropriate use of broad-spectrum antibiotics that may cause unnecessary and harmful side effects. In addition, our cases suggest that combination therapy with levocetirizine and hydroxyzine may be successfully used as corticosteroid-sparing treatment or to prevent relapse after the discontinuation of corticosteroid treatment. 
Cormerais et al.: Wells' Syndrome Mimicking Facial Cellulitis: A Report of Two Cases

\section{Statement of Ethics}

The authors state that the patients gave their informed consent. The research complies with all ethical guidelines for human studies.

\section{Disclosure Statement}

The authors declare no conflict of interest. They received no financial support.

\section{References}

1 Moossavi M, Mehregan DR: Wells' syndrome: a clinical and histopathologic review of seven cases. Int J Dermatol 2003;42:62-67.

-2 Wells GC: Recurrent granulomatous dermatitis with eosinophilia. Trans St Johns Hosp Dermatol Soc 1971;57:46-56.

3 Moon SH, Shin MK: Bullous eosinophilic cellulitis in a child treated with dapsone. Pediatr Dermatol 2013;30:e746-e747.

4 Spinelli M, Frigerio E, Cozzi A, Garutti C, Garavaglia MC, Altomare G: Bullous Wells' syndrome associated with non-Hodgkin's lymphocytic lymphoma. Acta Derm Venereol 2008;88:530-531.

5 Espana A, Sanz ML, Sola J, Gil P: Wells' syndrome (eosinophilic cellulitis): correlation between clinical activity, eosinophil levels, eosinophil cation protein and interleukin-5. Br J Dermatol 1999;140:127-130.

6 Ratzinger G, Zankl J, Zelger B: Wells syndrome and its relationship to Churg-Strauss syndrome. Int J Dermatol 2013;52:949-954.

7 Caputo R, Marzano AV, Vezzoli P, Lunardon L: Wells syndrome in adults and children. A report of 19 cases. Arch Dermatol 2006;142:1157-1161.

8 Sinno H, Lacroix JP, Lee J, Izadpanah A, Borsuk R, Watters K, Gilardino M: Diagnosis and management of eosinophilic cellulitis (Wells' syndrome): a case series and literature review. Can J Plast Surg 2012;20: 91-97.

9 Aroni K, Aivaliotis M, Liossi A, Davaris P: Eosinophilic cellulitis in a child successfully treated with cetirizine. Acta Derm Venereol 1999;79:332.

10 Thomson L, Blaylock MG, Sexton DW, Campbell A, Walsh GM: Cetirizine and levocetirizine inhibit eotaxininduced eosinophil transendothelial migration through human dermal or lung microvascular endothelial cells. Clin Exp Allergy 2002;32:1187-1192.

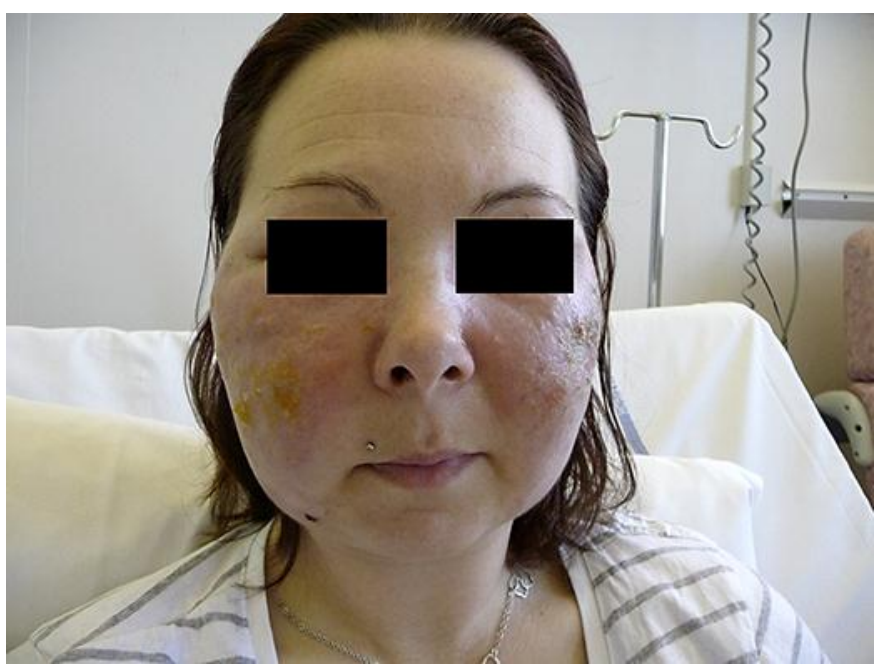

Fig. 1. Case 1. Facial erythematous edema covered by vesicles and yellowish crusts. 


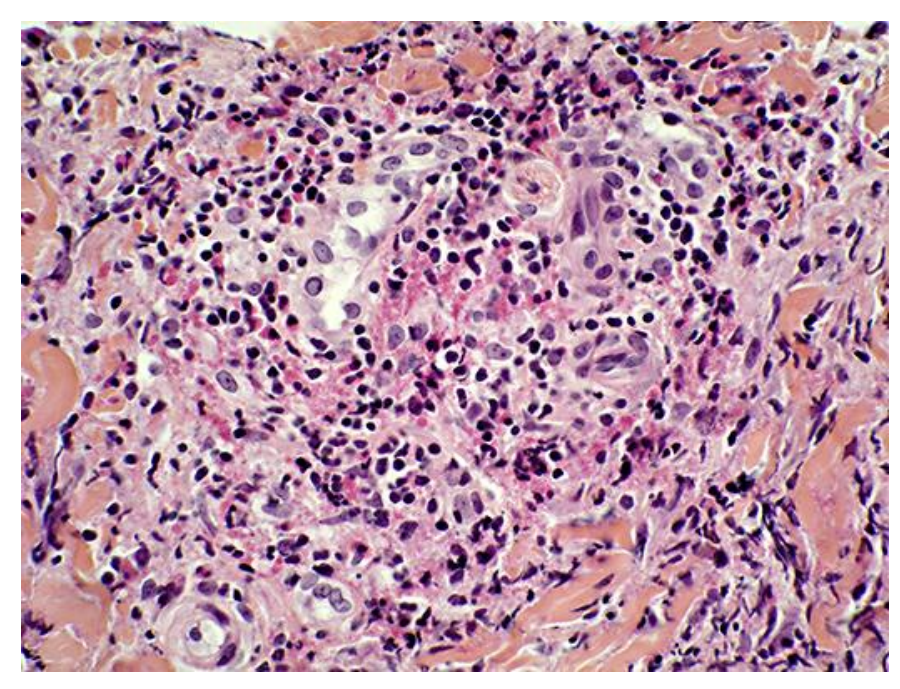

Fig. 2. Case 1. A skin biopsy specimen showing dense eosinophilic infiltration in the dermis, with no evidence of vasculitis. HE. $\times 40$.

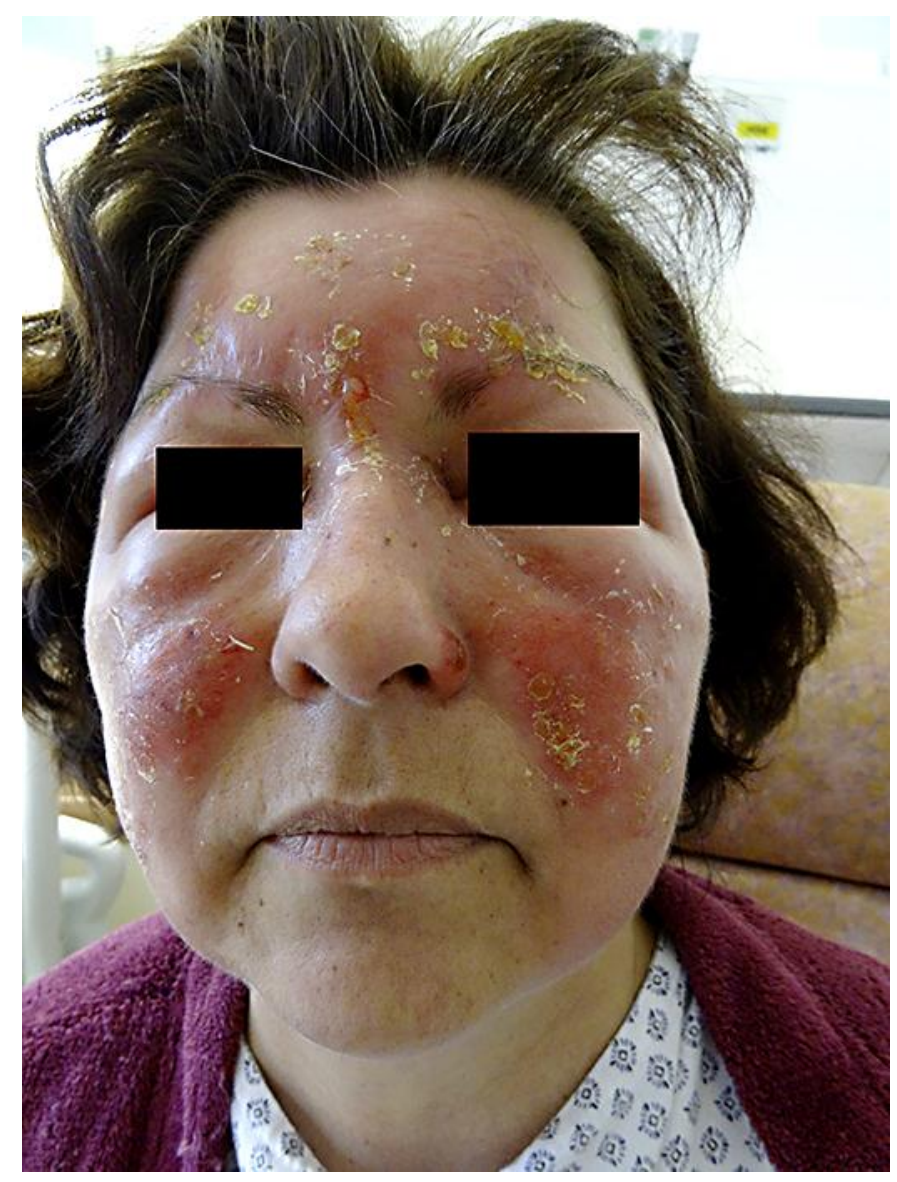

Fig. 3. Case 2. Facial erythematous edema with vesiculobullous lesions. 


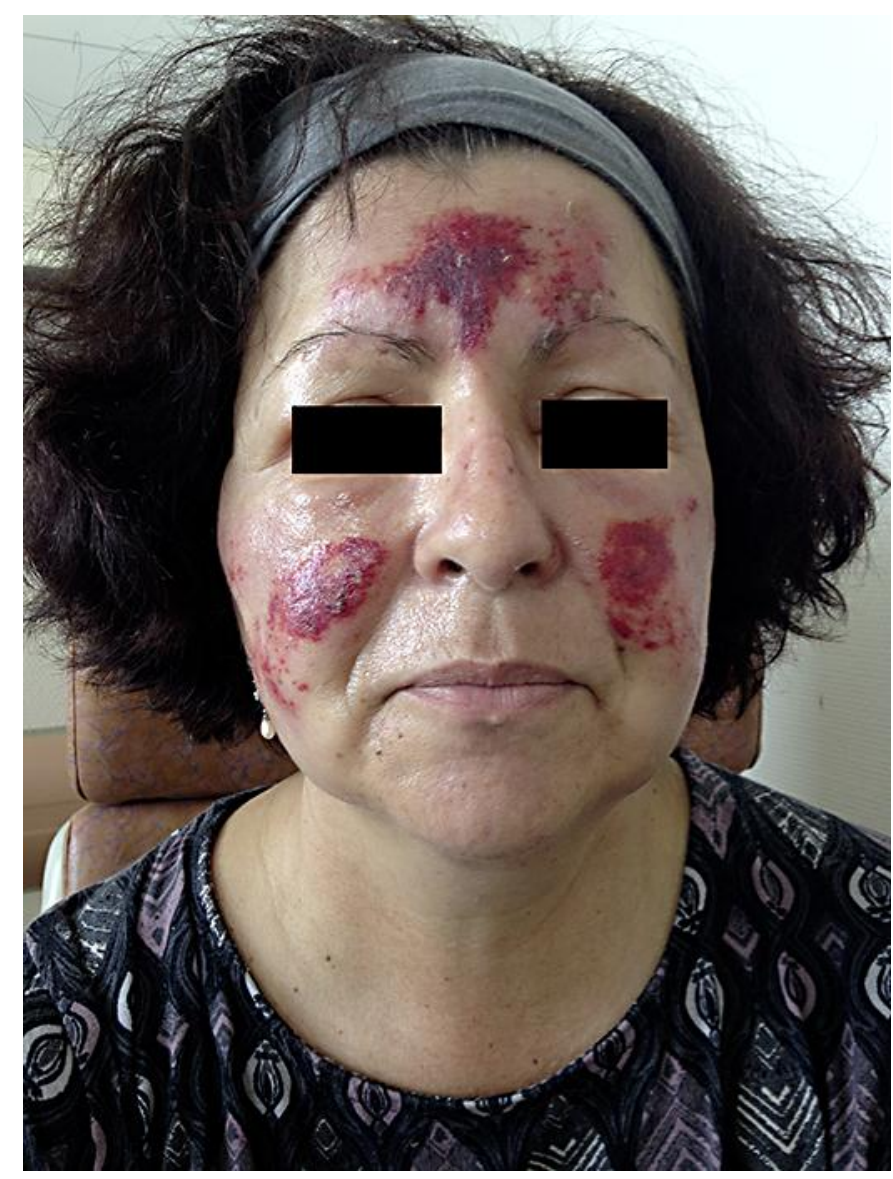

Fig. 4. Case 2. Dramatic improvement of skin lesions after 3 days of treatment with a combination therapy of levocetirizine and hydroxyzine. 